\title{
The Effect of Resin/Hardener Stoichiometry on the Electrical Properties of Silicon Nitride/Epoxy Nanocomposites
}

\author{
F. N. Alhabill, T. Andritsch and A. S. Vaughan \\ Tony Davies High Voltage Laboratory \\ University of Southampton, Southampton, UK \\ E-mail: fna1g13@soton.ac.uk
}

\begin{abstract}
The effect of resin/hardener stoichiometry was investigated for both unfilled epoxy and nanocomposite samples. The results indicate that incorporating silicon nitride nanofiller, which contains amine groups on its surface, has a significant influence on the resin/hardener reaction. At $2 \mathrm{wt} \%$ of nanofiller, it was estimated that the powder contains amine groups equivalent to around $5 \mathrm{wt} . \%$ of the hardener mass, which results in the displacement of the optimum resin/hardener mass ratio by the same amount $(\sim 5 \mathrm{wt} . \%)$. The dielectric spectra showed that the $\beta$ relaxation is directly related to the hydroxyether groups that are generated by the reaction between the epoxy and the amine groups. Therefore, the relaxation strength is proportional to the crosslinking density and consequently related to the glass transition temperature. The DC conductivity increases considerably as a result of incorporating silicon nitride nanofiller when not compensating for its impact on the resin/hardener stoichiometry. This might be related to the increase in the amine content of the material caused by the amine groups existing on the surface of the nanoparticles. When the stoichiometry effect is taken into account, the DC conductivity decreased to a value that is comparable to that of the unfilled polymer.
\end{abstract}

Keywords-nanocomposites; epoxy; silicon nitride; stoichiometry; glass transition temperature; dielecteric spectroscopy; DC conductivity;

\section{INTRODUCTION}

Research interest in utilizing nanotechnology for dielectric applications has grown immensely in the last decade. Many research groups worldwide have been conducting research to explore the potential of this technology to improve the performance of insulating materials and investigating the underlying mechanisms that control the behaviour of so-called nanodielectrics. It is widely accepted that the electrical performance of nanodielectrics is strongly influenced by the interactions that occur between the surface of the nanoparticles and the encapsulating polymer, i.e. within the interphase region [1]. These interactions are intensified by increasing the specific surface area of the particles, i.e. reducing the particle size, and by dispersing the particles efficiently within the matrix material $[2,3]$. However, if these interactions have a negative impact on dielectric performance, the effective dispersion will also aggravate this. Nanoparticle surface chemistry is an important factor that affects both interphase interactions and nanoparticle dispersion. Many studies have tried to modify the surface chemistry of nanoparticles before introducing them into the base polymer and some of these have reported improved electrical properties. However, the underlying mechanisms are not well understood, with different studies referred them to different factors, such as better particle dispersion [4], removal of polar moieties [5] and the introduction of electron traps [6].

For epoxy based nanocomposites, particles are incorporated into the resin before the curing process, which allows the particles to interact with the active as supplied resin or hardener. Any chemical reaction between the particles and the resin or the hardener will affect the reaction stoichiometry and thus result in a different network structure after curing.

Silicon nitride $\left(\mathrm{Si}_{3} \mathrm{~N}_{4}\right)$ is covered by amine and hydroxyl groups [7, 8]; both of these groups, particularly the amine, can react with the epoxy groups in the resin. Thus, this makes silicon nitride inherently compatible with an epoxy matrix and results in good particle dispersion, as can be observed from the scanning electron microscope images shown in a previous study [9]. The reaction between the amine groups on the surface of the particles and the epoxy groups will, however, reduce the epoxy groups available for reaction with the hardener, and consequently affect the stoichiometric ratio. Therefore, detecting an influence of the silicon nitride on the resin/hardener stoichiometry will be considered as evidence of reactions between the functional groups covering the surface of the silicon nitride nanoparticles and the epoxy groups from the resin.

This study therefore set out with the objective of investigating the effect of adding a silicon nitride nanofiller on the resin/hardener stoichiometry and the consequences of this for the electrical performance of the resulting materials.

\section{EXPERIMENTAL}

\section{A. Materials and samples preparation}

The silicon nitride $\left(\mathrm{Si}_{3} \mathrm{~N}_{4}\right)$ nanopowder utilised in this study was obtained from Sigma Aldrich. The supplier specifies a spherical shape and a particle size $<50 \mathrm{~nm}$. The epoxy resin used here was the dyiglycidyl ether of bisphenol-A (DGEBA) based resin, DER 332, obtained from Sigma Aldrich, and the hardener was the aliphatic diamine hardener, Jeffamine D 230, from Huntsman. The epoxy equivalent molar mass of DER 332 
is $\sim 174 \mathrm{~g} / \mathrm{mol}$ and the amine hydrogen equivalent molar mass of Jeffamine D230 is $60 \mathrm{~g} / \mathrm{mol}$. Thus, the theoretical resin:hardener stoichiometric ratio is 1000:344. Throughout this paper, the resin:hardener ratio is identified using a parameter termed the hardener percentage $(H P)$ which is defined by the following relation:

$$
H P=\frac{\text { mass of the hardener used }}{\text { hardener stoichoimetric mass }} \%
$$

where the hardener stoichiometric mass is the hardener mass following the stoichiometric ratio (344:1000). The samples are identified using a code consisting of three elements, the first refers to the epoxy resin, the second refers to the hardener percentage and the third refers to the nanofiller weight percentage (wt.\%). For example, E/100-H/2-SiN refers to an epoxy cured with $100 \%$ hardener percentage (stoichiometric ratio) and containing $2 \%$ silicon nitride; $\mathrm{E} / 80-\mathrm{H} / 0$ refers to epoxy cured with $80 \%$ hardener percentage without any filler. The unfilled epoxy sample, which employs the theoretical stoichiometric resin/hardener ratio (i.e. sample E/100-H/0), is taken as a reference for all other samples.

To prepare the unfilled epoxy sample, the required masses of the resin and the hardener were mixed using a magnetic stirrer for $15 \mathrm{~min}$ and then the mixture was degassed for $20 \mathrm{~min}$ before being injected into a metallic mould with an $\sim 200 \mu \mathrm{m}$ thick spacer. For nanocomposite samples, the filler was first dispersed into the resin using a probe sonicator for $45 \mathrm{~min}$ and then the mixture was heated at $100{ }^{\circ} \mathrm{C}$ for $3 \mathrm{~h}$, to stimulate any possible reaction, before the hardener was added and the above epoxy preparation procedure was followed. Based on the manufacturer's instructions, all samples were first cured in a fan oven at $80{ }^{\circ} \mathrm{C}$ for $2 \mathrm{~h}$ and then post-cured at $125^{\circ} \mathrm{C}$ for $3 \mathrm{~h}$.

\section{B. Differential Scanning Calorimetry}

The crosslink density of all sample was evaluated by determining the glass transition temperature $\left(T_{g}\right)$ using a Perkin Elmer DSC 7 differential scanning calorimeter (DSC). Two repeated DSC scans were performed for each material using specimen about $10 \mathrm{mg}$ in mass at a heating rate of $10{ }^{\circ} \mathrm{C} / \mathrm{min}$. $T_{g}$ was deduced from the second DSC scan, as the temperature corresponding to the maximum gradient of the heat capacity. The first scan was performed to erase the thermal history of the sample.

\section{Dielectric spectroscopy}

Dielectric spectroscopy was performed using a Solatron 1296 dielectric interface along with a Schlumberger SI 1260 impedance/phase gain analyser on specimens $30 \mathrm{~mm}$ in diameter. Before testing, each specimen was sputter coated with gold on both sides to provide a better sample/electrode contact. All measurements were performed at room temperature $\left(\sim 23^{\circ} \mathrm{C}\right)$ over the frequency range $0.1 \mathrm{~Hz}-1 \mathrm{MHz}$.

\section{DC Conductivity measurements}

DC conductivity was evaluated by measuring the current passing through the material of interest, using a Keithley 6487 picoammeter. The specimen was placed between opposing circular electrodes with a diameter of $20 \mathrm{~mm}$. Samples were sputtered coated with gold and stored dry in a vacuum desiccator before any measurements were made. However, there was no environmental control during the measurements process itself. All measurements were performed at a temperature of $30^{\circ} \mathrm{C}$ and an electric field of $42.5 \mathrm{kV} / \mathrm{mm}$.

\section{RESULTS AND DISCUSSION}

\section{A. The Glass Transition Temperature}

Figs. 1 and 2 respectively present DSC second scan data obtained from unfilled epoxy formulations and equivalent nanocomposites. From these traces, the $T_{g}$ was determined and the resulting data are presented in Fig. 3. For the unfilled epoxy samples, as expected, the maximum $T_{g}$ occurs at the theoretical stoichiometric ratio $(H P=100 \%)$. However, deviations to either side cause $T_{g}$ to decrease, but not in a totally symmetric way; initially, $T_{g}$ decreasing more sharply for the formulations with $H P<100 \%$ than for $H P>100 \%$. A comparable form of behaviour has been reported elsewhere $[10,11]$ for similar resin and hardener systems.

For the nanocomposite samples, a different trend can be observed. Here, $T_{g}$ does not decrease sharply for $H P<100 \%$, instead it decreases for $H P<90 \%$. Comparing the nanocomposite and the unfilled epoxy samples at an $H P$ value of $90 \%$ shows that $T_{g}$ for the samples containing the silicon nitride nanofiller is significantly higher. This implies that the effective amine groups : epoxy groups ratio is higher than $90 \%$ for the nanocomposite sample. Similarly, for sample E/100$\mathrm{H} / 2-\mathrm{SiN}$, it can be deduced that the actual hardener percentage is higher than $100 \%$, which explains the smooth decrease of $T_{g}$ compared with the equivalent unfilled sample (E/100-H/0). For sample $\mathrm{E} / 80-\mathrm{H} / 2-\mathrm{SiN}$, the 2 wt. $\% \mathrm{Si}_{3} \mathrm{~N}_{4}$ cannot compensate for the $20 \%$ reduction in the hardener and, consequently, its $T_{g}$ is significantly reduced. From these results, it can be deduced that $2 \mathrm{wt} . \%$ nano- $\mathrm{Si}_{3} \mathrm{~N}_{4}$ has an equivalent effect to around $5 \mathrm{wt} . \%$ of hardener. Fig. 4 shows $T_{g}$ results for all samples after shifting the HP 5 wt.\% to the right for the nanocomposite samples. Clearly, $T_{g}$ results obtained from the nanocomposite samples are equivalent to their counterparts from the unfilled samples, after increasing the $H P$ by $5 \%$, which reinforces the hypothesis adding 2 wt. $\%$ of nano- $\mathrm{Si}_{3} \mathrm{~N}_{4}$ is, from a reaction stoichiometry perspective, comparable to $5 \mathrm{wt} . \%$ of hardener.

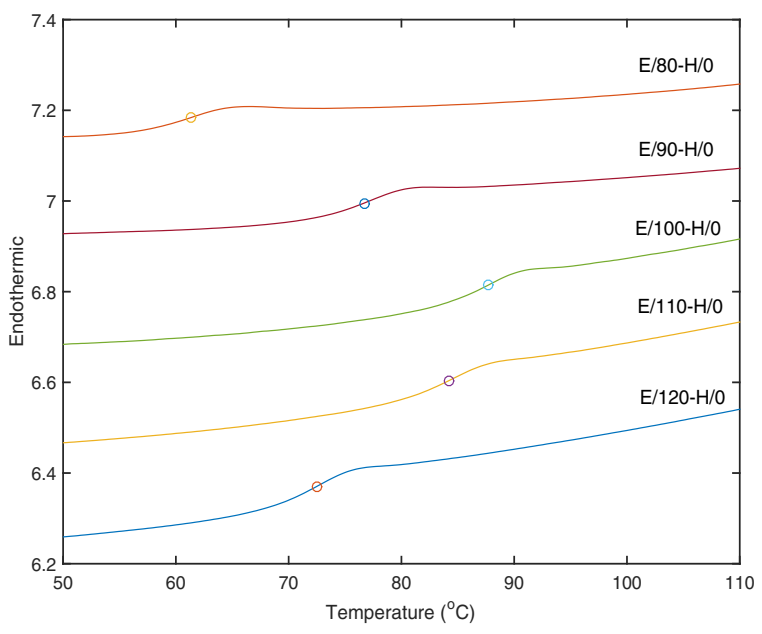

Fig. 1. DSC second scan traces obtained from unfilled epoxy samples with different formulations; the circles superimposed on the curves indicate $T_{g}$. 


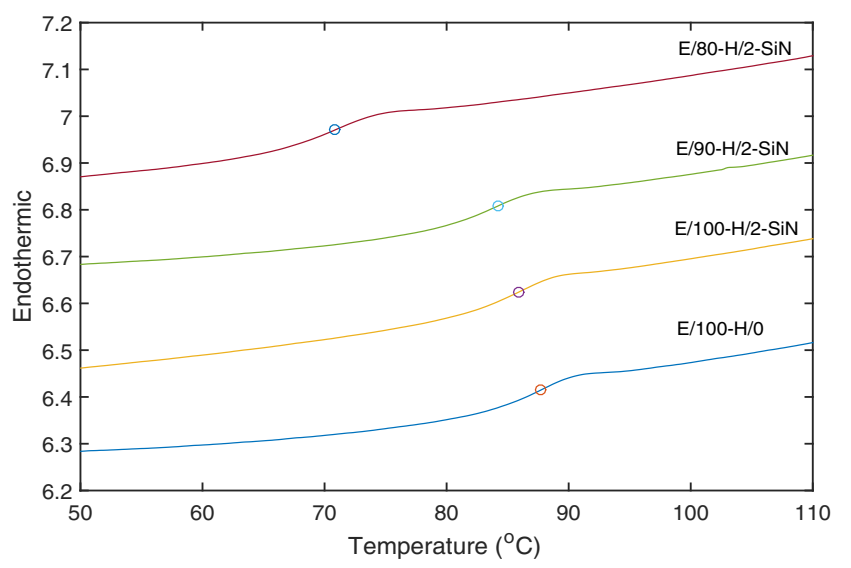

Fig. 2. DSC second scan traces obtained fron nanocomposite samples prepared with differenmt resin stoichiometries; the circles superimposed on the curves indicate $T_{g}$.

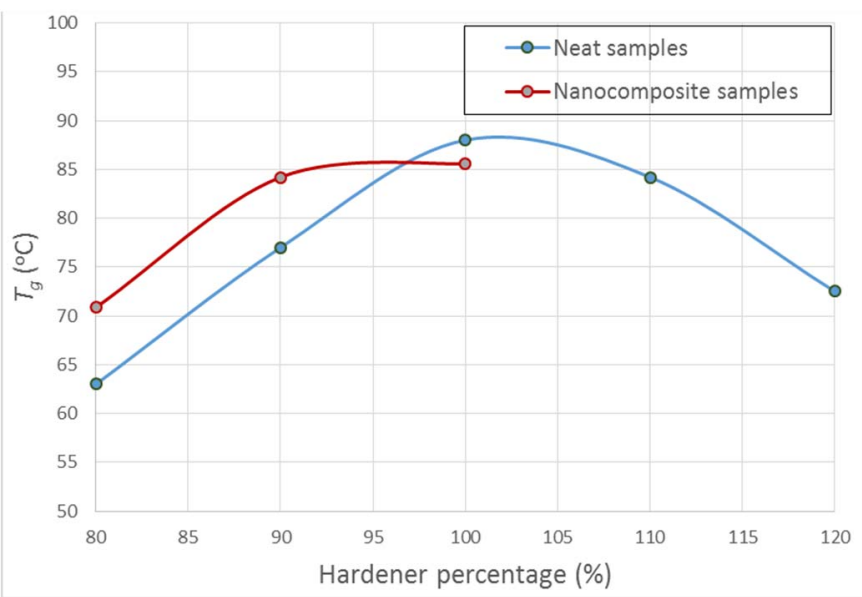

Fig. 3. The glass transition temperature for all samples.

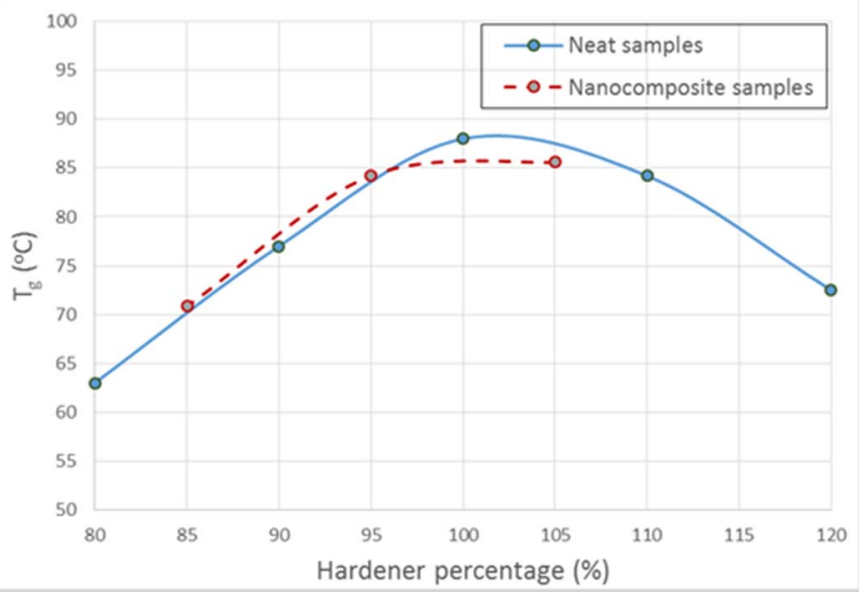

Fig. 4. The glass transition temperature for all samples after shifting the $H P$ $5 \%$ to the right for the nanocomposite samples.

\section{B. Dielectric spectroscopy}

Fig. 5 shows dielectric spectra obtained from nanocomposite samples, along with the reference sample $(\mathrm{E} / 100-\mathrm{H} / 0)$. A broad $\beta$ relaxation can be detected for all (a)

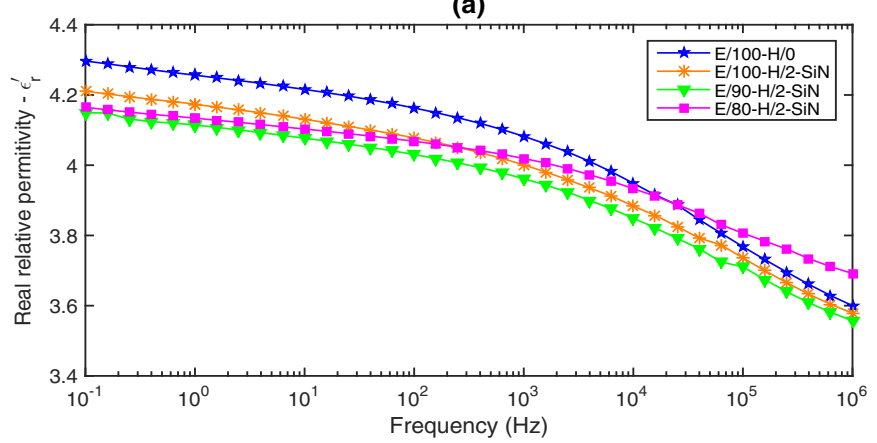

(b)

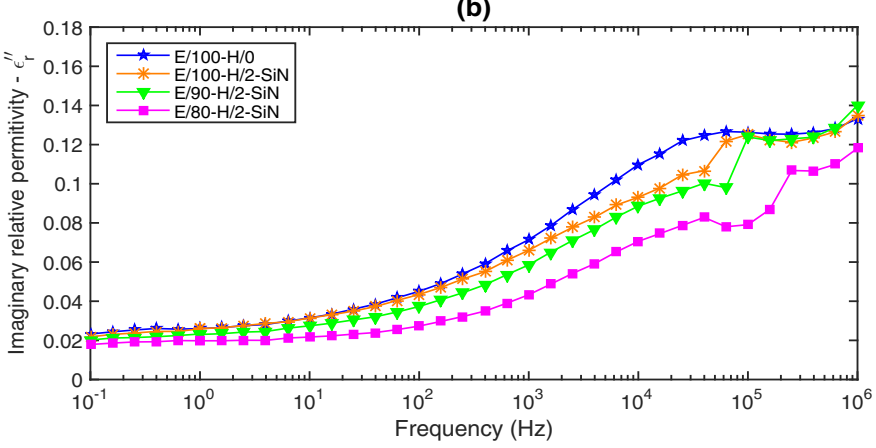

Fig. 5. Dielectric spectra for nanocomposites samples compared with the reference sample, (a) real permittivity and (b) imaginary permittivity.

samples, which peaks around $5 \times 10^{4} \mathrm{~Hz}$. This relaxation is associated with the crankshaft rotation of the hydroxyether group that is produced at each crosslink point by the reaction between epoxy and amine groups $[12,13]$. Thus, a higher crosslinking density implies a higher concentration of generated hydroxyether groups and, consequently, a stronger $\beta$ relaxation. Therefore, the $\beta$ relaxation is directly related to the crosslinking density of the network and consequently would be expected to correlate with the glass transition temperature. The imaginary permittivity, Fig. 5 (b), shows this relation where the relaxation strength decreases for the samples with lower $T_{g}$ and vice versa. The nanofiller does not have a noticeable influence on the dielectric spectra over the frequency range shown at the measurement temperature.

\section{DC Conductivity}

The effect of the nanofiller and the different resin/hardener ratios on charge mobility was investigated by measuring the DC conductivity of the nanocomposite samples along with the reference unfilled epoxy. The resulting data are presented in Fig. 6 and show that sample E/100-H/2-SiN has a considerably higher DC conductivity compared with the other samples. This might be related to its higher amine content, where the effective hardener percentage for this sample is expected to be greater than $100 \%$, due to consumption of epoxy groups through reaction with amine groups on the surface of the nanoparticles. The other nanocomposite samples exhibit a slight increase in the DC conductivity compared with the unfilled epoxy, see Table 1. However, the data for these three samples are overlapping and within experimental uncertainties. 


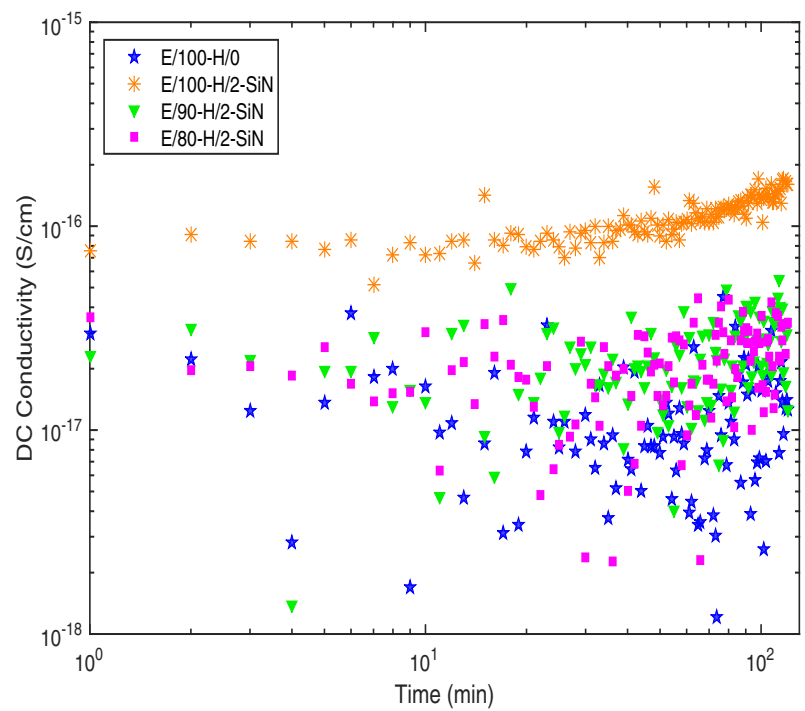

Fig. 6. DC conductivity for nanocomposite samples compared with the reference sample.

TABLE I. DC CONDUCTIVITY AVERAGED OVER 2 HOURS

\begin{tabular}{|l|l|l|l|l|}
\hline Sample code & E/100-H/0 & E/100-H/2-SiN & E/90-H/2-SiN & E/80-H/2-SiN \\
\hline $\begin{array}{l}\text { DC conductivity } \\
\text { (S/cm) }\end{array}$ & $1.0 \times 10^{-17}$ & $11.1 \times 10^{-17}$ & $2.1 \times 10^{-17}$ & $1.7 \times 10^{-17}$ \\
\hline
\end{tabular}

The variation of DC conduction current with time is generally expected to take the form $I=A \mathrm{e}^{-b t}[14]$. However, from Fig. 6, it is evident that the behaviour of $\mathrm{E} / 100-\mathrm{H} / 2-\mathrm{SiN}$ is very different from this in that a clear increase in conductivity with time is evident beyond $\sim 60 \mathrm{~min}$. Comparable behaviour has been seen elsewhere and discussed in terms of two processes: trap filling effects [15]; absorption of water influencing charge injection from electrodes [16]. The origin of the process shown here is currently unclear, but an investigation into the influence of water on charge transport dynamics in epoxy/silicon nitride nanocomposites is currently underway.

\section{CONCLUSION}

The interactions between a nanofiller and the base polymer can significantly affect the performance of nanocomposite systems. Particularly for nanocomposites based on thermosetting polymers, chemical interactions between the nanoparticles and either the resin or the hardener could change the reaction stoichiometry and, therefore, result in the development of different network structures. Thus, particle dispersion is not the only factor which affects the performance of the nanocomposites. Other factors like the chemical content could affect the electrical performance considerably, as was observed by the increase in the DC conductivity caused by the excess amine group resulting from introducing the silicon nitride nanofiller.

\section{REFERENCES}

[1] M. Roy, J. K. Nelson, C. W. Reed, R. K. MacCrone, R. J. Keefe, W. Zenger, et al., "Polymer nanocomposite dielectrics - The role of the interface (vol 12, pg 629, 2005)," IEEE Transactions on Dielectrics and Electrical Insulation, vol. 12, pp. 1273-1273, Dec 2005.

[2] J. Y. Li, L. Zhang, and S. Ducharme, "Electric energy density of dielectric nanocomposites," Applied Physics Letters, vol. 90, p. 132901, 2007.

[3] M. Praeger, T. Andritsch, S. G. Swingler, and A. S. Vaughan, "A simple theoretical model for the bulk properties of nanocomposite materials," in 2014 IEEE Conference on Electrical Insulation and Dielectric Phenomena, ed, 2014, pp. 699-702.

[4] C. Yeung, and A. S. Vaughan, "A study of how varying degrees of functionalised nanofiller have an effect on nanodielectrics," 2012 Annual Report Conference on Electrical Insulation and Dielectric Phenomena (Ceidp), pp. 319-322, 20122012.

[5] M. Praeger, I. L. Hosier, A. S. Vaughan, and S. G. Swingler, The effects of surface hydroxyl groups in polyethylene-silica nanocomposites, 2014.

[6] S. Virtanen, T. M. Krentz, J. K. Nelson, L. S. Schadler, M. Bell, B. Benicewicz, et al., "Dielectric breakdown strength of epoxy bimodalpolymer-brush-grafted core functionalized silica nanocomposites," IEEE Transactions on Dielectrics and Electrical Insulation, vol. 21, pp. 563570, Apr 2014.

[7] B. Fubini, M. Volante, V. Bolis, and E. Giamello, "Reactivity towards water of silicon nitride: energy of interaction and hydration dehydration mechanism " Journal of Materials Science, vol. 24, pp. 549-556, 1989.

[8] J. Szepvolgyi, I. Mohai, and J. Gubicza, "Studies on atmospheric ageing of nanosized silicon nitride powders," Key Engineering Materials, vol. 264-268, pp. 2311-2314, 2004.

[9] F. N. Alhabill, T. Andritsch, and A. S. Vaughan, "Effect of the Processing Method on the Electrical Behavior of Silicon Nitride/Epoxy Nanocomposites," in 2015 IEEE Conference on Electrical Insulation and Dielectric Phenomena, ed, 2015, pp. 511-514.

[10] F. Meyer, G. Sanz, A. Eceiza, I. Mondragon, and J. Mijovic, "The effect of stoichiometry and thermal history during cure on structure and properties of epoxy networks," Polymer, vol. 36, pp. 1407-1414, Mar 1995.

[11] R. J. Morgan, F.-M. Kong, and C. M. Walkup, "Structure-property relations of polyethertriamine-cured bisphenol-a-diglycidyl ether epoxies," Polymer, vol. 25, pp. 375-386, 1984.

[12] C. Jordan, J. Galy, and J. P. Pascault, "Measurement of the extent of reaction of an epoxy-cycloaliphatic amine system and influence of the extent of reaction on its dynamic and static mechanical-properties," Journal of Applied Polymer Science, vol. 46, pp. 859-871, Oct 151992.

[13] C. L. Soles and A. F. Yee, "A discussion of the molecular mechanisms of moisture transport in epoxy resins," Journal of Polymer Science Part B-Polymer Physics, vol. 38, pp. 792-802, Mar 12000.

[14] T. C. Guo and W. W. Guo, "A transient-state theory of dielectricrelaxation and the curie-vonschweidler law," Journal of Physics C-Solid State Physics, vol. 16, pp. 1955-1960, 1983.

[15] K. Y. Lau, A. S. Vaughan, G. Chen, I. L. Hosier, K. Y. Ching and N. Quirke, "Polyethylene/silica nanocomposites: absorption current and the interpretation of SCLC", submitted to J. Phys D: Appl. Phys.

[16] M. Praeger, I. L. Hosier, A. F. Holt, A. S. Vaughan and S. G. Swingler, "On the Effect of Functionalizer Chain Length and Water Content in Polyethylene/Silica Nanocomposites: Part II - Charge Transport", submitted to IEEE Trans. Diel. Electr. Insul. 